\title{
$H$-adaptive mesh refinement for Topology Optimization in the framework of the Cartesian grid finite element method - cgFEM
}

\author{
D. Muñoz, J. Albelda, J.J. Ródenas, E. Nadal \\ Instituto de Investigación en Ingeniería Mecánica y Biomecánica (I2MB) \\ Universitat Politècnica de València - Spain \\ e-mail: damuopel@upv.es, \{jalbelda, jjrodena\}@mcm.upv.es, ennaso@upvnet.upv.es
}

\begin{abstract}
The growing interest in Topological Optimization (TO) in recent years is undeniable. In particular, this paper focuses on the Solid Isotropic Material Penalization (SIMP) method [1], broadly used because of its simple formulation and efficiency, and how $h$-adaptive mesh refinement in the context of the Cartesian grid finite element method, $c g$ FEM [2], can improve its performance.

This paper will present a combination of three methodologies based on mesh $h$-adaptation in $c g$ FEM (whose hierarchical mesh and data structures are specially efficient in TO) to overcome two disadvantages of the SIMP method related to the lack of sharpness in the definition of the boundary and the influence of the finite element (FE) discretization on the final solution:
\end{abstract}

- Use of two mesh levels: i) a mesh for the FE analysis and ii) a refined mesh, not only for integration and calculation of sensitivities but also to enhance sharpness of the boundary representation at a marginal computational cost.

- Use a density-based refinement: a sharper boundary definition will be obtained using a basic technique where elements with intermediate density values are refined.

- Use of solution-based refinement: the numerical solution obtained by the use of a $h$-adaptive analysis technique based on a recovery-type error estimator guaranties that an accurate evaluation of stress values is considered in stress-constrained TO.

Acknowledgements The authors appreciate the financial support to this work of the Spanish Ministerio de Economa, Industria y Competitividad (DPI2017-89816-R) and the Ministerio de Educación (FPU16/07121).

\section{REFERENCES}

[1] O. Sigmund. A 99 line topology optimization code written in Matlab. Structural and Multidisciplinary Optimization. 21:120-127, 2001.

[2] E. Nadal. Cartesian grid FEM (cgFEM): High performance h-adaptive FE analysis with efficient error control: application to structural shape optimization. Ph.D. Thesis, Universitat Politècnica de València, 2014. 